\title{
Visual recognition: Facilitation of seeing by hearing'
}

William M. Smith DARTMOUTH COLLEGE 2

\begin{abstract}
A previous finding of enhanced visual recognition through vocalization by $\mathrm{S}$ is confirmed and extended by the present experiment which shows that similar effects on visual recognition occur when a voice other than S's is employed. It is concluded that such facilitation of visual recognition is the result of perceptual interaction, not kinesthetic or auditory feedback, as such, nor factors of expectancy and set.
\end{abstract}

\section{Problem}

In a recent paper (Smith, 1965) it was reported that visual recognition was enhanced when S's voice was used to trigger tachistoscopic exposures of words which matched the words spoken. It was concluded that this finding was open to alternative interpretations. One suggested interpretation concerned auditory or kinesthetic feedback or both. Because the visual exposures occurred early in the period of vocalization, $S$ obtained both auditory and kinesthetic feedback slightly before, during and after the completion of the visual exposure. An interaction of such feedback information with the congruent visual stimulation, therefore, might enhance recognition. Another possible interpretation related to expectancy or set. Prior to each visual exposure $\mathrm{E}$ told $\mathrm{S}$ which word he was to vocalize on that trial, and, therefore, $\mathrm{S}$ had to remember for a brief period what the word was, and then say it. Consequently, it could be argued that the transitory memory of and set to say a particular word might in some way sensitize or "prime" recognition in those instances where set matched the word exposed. Finally, it was stated that perceptual interaction, independent of that associated with feedback as noted above, could account for the increased recognition. That is, perhaps the mere fact of hearing a word while being exposed to it visually would be sufficient to enhance recognition.

The present experiment was designed for the purpose of eliminating one or more of these alternative explanations by comparing recognition under two contrasting conditions, one using $S$ 's voice and the other using $\mathrm{E}^{\prime} \mathrm{s}$ voice.

\section{Method}

A pilot experiment performed prior to the experiment reported here demonstrated the same facilitation of visual recognition for numbers as had been found in the earlier experiments on words (Smith, 1965). In the present experiment, therefore, two-digit numbers (21-99) excepting 30,40 , et cetera, were employed.

The general procedure was the same as that described in the earlier report (Smith, 1965). Lists of 25 two-digit numbers were used. The numbers were exposed in a Scientific Prototype 3-channel Tachisto- scope, black letters on a white background. Each S in each experimental condition was given different lists of numbers, both vocalized and presented. Moreover, the numbers chosen for a match between vocalization and visual presentation differed for each $\mathrm{S}$ in each condition.

The two major experimental conditions consisted of "own voice" (OV) and "E voice" (EV). In OV the procedure was exactly the same as in the experiments with words. In $\mathrm{EV}$ an $\mathrm{E}$ sat in an adjacent room and spoke the numbers over an intercom system. A speaker located next to Senabled him to hear clearly the numbers $\mathrm{E}$ vocalized. Vocalization of a number by $\mathrm{E}$ activated a voice key which in turn triggered the visual exposure of a number.

The 25 trials in each of OV and EV were arranged in blocks of five trials. Each block of five contained 0 , 1 , or 2 matched presentations (M), that is, the number spoken by $\mathrm{E}$ was the same as the number exposed. A total of five out of 25 trials were matched. Matching was done on a random basis so that no particular number or numbers were favored. Sixteen S's were used, half of whom were given OV first, half EV first. All Ss possessed normal acuity as measured by an Ortho-rater.

Preliminary thresholds for each of three numbers were obtained for each S using a method of limits. The median estimate was employed as the exposure duration for that $\mathrm{S}$ in the experiment. On each trial $\mathrm{S}$ was given a ready signal to fixate properly, after which a number was uttered by $\mathrm{E}$, and $\mathrm{S}$ reported his response. Instructions to $\mathrm{S}$ were to respond when he thought he had at least a 50 percent chance of being correct. The brightness of the pre-exposure field and the test field was approximately $.2 \mathrm{ft}$ lamberts. The delay between the beginning of vocalization and the visual exposure was approximately $20 \mathrm{msec}$, hence the visual exposure occurred in the early part of vocalization and well before the end of it. Exposure durations for the $16 \mathrm{Ss}$ varied between 30 and $140 \mathrm{msec}$, only one of which exceeded $100 \mathrm{msec}$. Nine of the 16 exposure durations were $50 \mathrm{msec}$. or less.

\section{Results}

For each $\mathrm{S}$ in $\mathrm{OV}$ and $\mathrm{EV}$ the percent correct responses for the matched trials (M) and the mismatched trials (MM) were computed. In order to correct for the possible tendency for S to say what he heard (or said) and thus increase the probability of a "correct" response on a matched trial, the mismatched data were used to correct the matched data. This correction was made in $\mathrm{OV}$ and $\mathrm{EV}$ for each $\mathrm{S}$ by determining the proportion of times that $S$ responded on a mismatched trial with a number the same as he had just said, or had just heard 
E say. The correction ignored the "no-response" mismatched trials, and was, therefore, the most stringent correction possible. Data was corrected by this procedure for 10 of the $16 \mathrm{Ss}$.

The mean percent correct responses for OV vs. EV and within each, M vs. MM, suggest what statistical analysis confirms. The mean percent correct under $M$ for $O V$ and $E V$ were 54 and 59, respectively. The corresponding percentages under MM were 37 and 45 . A three-way analysis of variance revealed no significant interactions nor a reliable difference between $\mathrm{OV}$ and EV $(F=3.23$ with 1 and $15 \mathrm{df}$ ). Variance due to Ss was significant, as was that for the M-MM comparison ( $F=17.09$ with 1 and $15 \mathrm{df} ; \mathrm{p}=<.001)$.

\section{Diseussion}

The fact that the results show no reliable difference between $\mathrm{OV}$ and $\mathrm{EV}$, but a highly reliable difference between $\mathbf{M}$ and $\mathbf{M M}$ (better recognition in favor of $M$ ) confirms the findings of the earlier experiments (Smith, 1965), and provides a basis for rejecting two of the three alternative interpretations offered for those earlier results. Present results support the interpretation that the facilitation of recognition found is due to a perceptual interaction effect, namely, an effect of hearing on seeing.
Having $\mathrm{E}$ vocalize precludes the possibility that the facilitory effect is due to a factor of set or expectancy because in such circumstances $S$ has no way of expecting or getting set for a specific word or number, as he could if he repeated what $\mathrm{E}$ had just instructed him to say. Moreover, having $\mathrm{E}$ rather than $\mathrm{S}$ vocalize a word or number eliminates feedback as such as a possible explanation of the facilitation. What remains as an explanation is, therefore, hearing while seeing. The S may hear his own voice or that of $\mathrm{E}$; in either case his visual recognition will be enhanced if what he hears is congruent with what is exposed visually.

Finally, because of the fact that in these experiments visual exposure occurred well before $S$ heard the complete word or number, some kind of short-term memory mechanism bridging vision and audition is implicated.

Reference

SMITH, WILLIAM M., Visual recognition: Facilitation of seeing by saying. Psychon. Sci., 1965, 2, 57-58.

Notes

1. This work was supported in part by National Science Foundation Grant GB-1110. I thank S. Jackson for her assistance.

2. On leave, 1964-65, California Institute of Technology and University of California, Los Angeles. 